\title{
Multiple Drug Intolerance Syndrome: A Large-Scale Retrospective Study
}

\author{
Hisham M. R. B. Omer • James Hodson • \\ Sarah K. Thomas · Jamie J. Coleman
}

Published online: 2 November 2014

(C) The Author(s) 2014. This article is published with open access at Springerlink.com

\begin{abstract}
Background The term multiple drug intolerance syndrome (MDIS) has been used to describe patients who express adverse drug reactions to three or more drugs without a known immunological mechanism.

Objective To identify patient factors that could increase the risk of MDIS.

Method Inpatient records over a 5-year period were captured from an electronic prescribing system to identify patients with at least one documented drug allergy. Univariable and multivariable analyses were used to compare the rates of MDIS across age, sex, weight, ethnicity, history of atopy or psychological disorders, and previous admissions.

Results A total of 25,695 patients had a documented drug intolerance, $4.9 \%$ of whom had MDIS. MDIS was significantly more likely in women $(p<0.001)$, patients with multiple comorbidities $(p<0.001)$, and patients with previous hospital admissions $(p<0.001)$. With the exception of penicillin $(p=0.749)$, MDIS was more frequent in those with allergies to other drugs $(p<0.001)$.

Conclusion MDIS was associated with female gender, multiple comorbidities, and previous hospital admissions. A documented allergy to penicillin did not increase the likelihood of MDIS.
\end{abstract}

H. M. R. B. Omer · S. K. Thomas ( $₫)$ · J. J. Coleman College of Medical and Dental Sciences, University of Birmingham, Edgbaston, Birmingham B15 2TT, UK e-mail: s.k.thomas@bham.ac.uk

J. J. Coleman

e-mail: j.j.coleman@bham.ac.uk

J. Hodson · S. K. Thomas · J. J. Coleman

University Hospitals Birmingham NHS Foundation Trust, Birmingham, UK

\section{Key Points}

Multiple drug intolerance syndrome (MDIS) is significantly more likely in female patients, patients with comorbidities, and patients with previous hospital admissions

Deprivation and ethnicity are not significant risk factors for MDIS

With the exception of penicillin, allergies to a broad spectrum of drugs including nonpenicillin antibiotics are identified as significant risk factors for MDIS

\section{Introduction}

Adverse drug reactions are not uncommon, and ascertaining the allergy status of a patient is an important part of the history taking process. A missed or incorrect diagnosis can have serious or even fatal consequences. Drug allergy is defined by the British Society for Allergy and Clinical Immunology as an adverse drug reaction with an established immunological mechanism [1]. In contrast, other adverse drug reactions not caused by an immunological mechanism may be pseudo-allergic, idiosyncratic, or defined as an intolerance [2]. The term multiple drug intolerance syndrome (MDIS) has been used to describe patients who express adverse drug reactions to three or more drugs without a known immunological mechanism [3]. The prevalence of MDIS in the UK is unknown, although a large study in the USA found that $2.1 \%$ of 
patients enrolled in a health plan group had three or more drug intolerances [4]. In spite of the seemingly low prevalence of MDIS, these cases pose a real problem for physicians. Often the fear of exacerbating an illness or triggering an anaphylactic reaction means that physicians avoid the list of culprit drugs at all costs. This can complicate treatment plans through the inability to prescribe optimal first-line therapies and necessitates the use of alternative, possibly less-effective treatments [5]. This, as well as variations in care, management, and diagnosis of drug allergy in the UK, has led to the provision of guidance from the National Institute for Health and Care Excellence [2].

The mechanisms underlining MDIS are not well understood, but some researchers have proposed the idea of nonspecific histamine release by mast cells and basophils [6]. It has recently been shown that MDIS patients have a strong wheal-and-flare response to autologous serum [7]. This suggests the presence of autoreactive antibodies in the serum of patients with MDIS. It is thought that these antibodies, when triggered by culprit drugs, may target the high affinity $\mathrm{IgE}$ receptor (FceRI) to induce histamine release. However, preliminary results have shown that sera from MDIS patients are unable to stimulate significant histamine release from donor basophils. Whether this mechanism truly underpins the pathogenesis of MDIS requires further clarification.

Psychological factors may also have a role to play in MDIS. Evidence suggests that MDIS patients have higher levels of anxiety, worse health-related quality-of-life scores, and increased likelihood of somatisation [8,9]. Such factors may have a link to the nocebo effect, which is defined as the emergence of negative effects following exposure to a nonharmful substance [10]. Patients who have experienced a reaction to a drug are likely to have negative thoughts associated with that drug. In addition, patients are prone to elevated anxiety levels prior to elective procedures. These negative thoughts are likely to have an influence on the subjective symptoms reported by MDIS patients to culprit drugs. As a result, physicians may struggle to differentiate between symptoms attributed to somatisation and those attributed to drug allergies [9].

In the present study, we investigated the characteristics of patients with three or more documented drug intolerances, and compared these to patients with one or two intolerances in order to determine whether any patient factors are associated with MDIS. For the purpose of this study, we refer to all patient-reported adverse effects as "allergy". Quotes are used to signify that patient-reported "allergies" do not necessarily represent true type I hypersensitivity reactions. The proportion of patients whose reactions are strictly $\operatorname{IgE}$ mediated in nature is unknown.

\section{Methods}

\subsection{Setting}

This work was carried out in a large acute NHS Foundation Trust. The Trust has a locally developed electronic prescribing and administration system known as PICS (prescribing, information and communication system), which is used for prescribing and documenting the administration of medicines throughout all $(\sim 1,200)$ inpatient beds, as well as capturing information required to aid this process, such as the allergy status of a patient. The system was first installed in the renal unit in 1998 [11], and now covers all general and specialist medical and surgical specialities. A key feature of the system, for the purposes of this study, is that on a weekly basis all information within the system is exported to a comprehensive audit database for subsequent investigation and analyses.

\subsection{Data Collection}

Inpatient episodes between 1 January 2009 and 31 July 2013 that had a documented allergy were captured for analysis. For each patient, the demographics and medical and drug histories were captured from PICS for further analysis (Box 1).

Box 1 Patient and medical information captured on PICS for each inpatient episode with a documented allergy

\begin{tabular}{ll}
\hline Patient demographics & Age \\
& Sex \\
& Ethnicity \\
& Weight \\
& Post-code \\
& Drug name \\
Allergies & Number of documented comorbidities \\
Medical history & Number of previous admissions to the Trust: \\
& atopic history: asthma (ICD10; J45), \\
& conjunctivitis (H10), eczema (B00.0) and \\
& atopic dermatitis (L20), dermatitis (L23- \\
& L27), rhinitis (J30), psoriasis (L40 \& L41) \\
& Psychological comorbidities: \\
& schizophrenia and other psychoses \\
& (F20-F29); affective disorders (F30-F39); \\
& neuroses stress-related and somatoform \\
& disorders (F40-F48); behavioural and \\
& personality disorders (F90-F98); other \\
& organic disorders (F00-F09) \\
& Presence of a prescription for: \\
& Antihistamines \\
& Adrenaline 1:1,000 (or preparations of this \\
& such as Epipen ${ }^{\circledR}$ ) \\
& Corticosteroids: prednisolone, hydrocortisone \\
&
\end{tabular}




\subsection{Data Analysis}

Individual drug allergy entries were categorised as antibiotics or nonantibiotics. The antibiotics were then further divided according to frequency of occurrence into eight categories: cephalosporins, glycopeptides, macrolides, penicillins, quinolones, tetracyclines, trimethoprim/sulfonamides, and other antibiotics.

The nonantibiotic group were also further divided according to frequency of occurrence into 11 categories: angiotensin converting enzyme inhibitors (ACEi), antihistamines, aspirin, latex, lipid regulators, nonsteroidal antiinflammatory drugs (NSAIDs), opioids, paracetamol, peanuts, shellfish, and other nonantibiotics. The "other nonantibiotics" class contained less commonly reported drug allergies such as anticoagulants, antiemetics, antihypertensives, and antimuscarinics. Peanuts and shellfish were included in the data extraction owing to their use in some dietary supplements and prescription medicines.

Patients were categorised into one of two groups: the MDIS group (defined as the recording of three or more drug allergies) or the non-MDIS group (patients with one or two reported drug allergies). For the analysis of demographic factors, patients with missing or clearly spurious data (e.g., impossibly low body weight) were excluded. In cases where patients had multiple admissions during the time period, the mean weight was calculated. Patient age was taken at the first record of a documented drug allergy.

Patient postcodes were attributed to lower level super output areas (LSOAs) using the national look-up file maintained by the Office for National Statistics [12, 13]. For each postcode-derived LSOA, income deprivation was calculated using the Indices of Deprivation 2007 income domain score [14]. Income deprivation is defined as the proportion of people earning $65 \%$ or less of the median English household income [15]. Postcodes that could not be attributed an LSOA were excluded from further analysis (for example, British forces post office codes and ZZ codes for patients outside the UK/or who have no fixed abode or temporary residence).

\subsection{Statistical Analysis}

Initially, Mann-Whitney and Fisher's exact tests were used to perform univariable analysis of the association between MDIS and a range of demographic factors. This was then extended to a multivariable binary logistic regression model in order to consider these factors simultaneously.

The next stage of the analysis considered how MDIS rates differed in patients prescribed selected antiallergic agents, and those with psychological or atopic comorbidities. Univariable analysis was performed with Fisher's exact tests, which were then followed by a multivariable binary logistic regression model. This model also included all of the demographic factors analysed previously, in order to account for known associations with MDIS.

The final stage of the analysis used univariable Fisher's exact tests to determine the drug classes that were most influential in predicting the patients with MDIS. All analyses were performed using IBM SPSS v22 (IBM SPSS Inc., Armonk, NY, USA) with statistical significance assessed at the $5 \%$ level.

\section{Results}

Between 1 January 2009 and 31 July 2013 there were 25,695 patients admitted on PICS with at least one documented drug allergy. A total of 1,250 (4.9\%) had three or more drug allergies and were categorised as having MDIS.

A univariable analysis showed that age was significantly greater (median $=60$ vs. 56 years, $p<0.001$ ) and weight was significantly lower (median $=71.2$ vs. $74.0 \mathrm{~kg}$, $p<0.001$ ) in MDIS patients (see Table 1). The analysis also showed that females were approximately twice as likely to be classed as multiple drug intolerant (6.1 vs. $2.9 \%, p<0.001)$. Deprivation scores did not differ significantly between the groups (both medians $=0.17$, $p=0.214)$ and there was no significant difference across different ethnic groups $(p=0.163)$. MDIS cases were significantly more frequent in patients with more comorbidities $(p<0.001)$, increasing from $3.5 \%$ for those with no comorbidities to 4.8 and $7.3 \%$ for those with one and multiple comorbidities, respectively. Similarly, the frequency of MDIS was significantly greater $(p<0.001)$ in patients with at least one hospital admission prior to the recording of their first drug allergy (5.3\%) compared to those with no previous hospital admissions (3.2\%).

The effects of population demographics were also assessed using multivariable analysis (see Table 2). Odds ratios (ORs) were expressed for each factor as predictors for multiple drug intolerance. The analysis showed that the presence of comorbidities was a significant predictor of MDIS $(p<0.001)$. ORs were significantly greater for patients with two or more comorbidities [OR 1.91, $95 \%$ confidence interval (CI) $1.64-2.22, p<0.001$ ] or with a single comorbidity (OR 1.26, $95 \%$ CI 1.05-1.52, $p=0.012$ ) than in patients with none. A history of admissions prior to the first recorded drug allergy was also a significant predictor of MDIS (OR 1.59, $95 \%$ CI $1.30-1.94, p<0.001)$ compared to no previous admissions. Sex remained significant, with women significantly more likely to have MDIS (OR 2.06, $95 \%$ CI 1.77-2.40, $p<0.001$ ).

After accounting for the effects of these factors, neither age $(p=0.716)$ nor weight $(p=0.364)$ was found to be 
Table 1 Univariable analysis of the effects of demographic factors on rates of multiple drug intolerance

\begin{tabular}{lccc}
\hline Factor & \multicolumn{2}{c}{ Multiple drug intolerance } & \multirow{2}{*}{$p$ value } \\
\cline { 2 - 3 } & No & Yes & \\
\hline Age (years) & $56(40,71)$ & $60(44,73)$ & $<0.001^{*}$ \\
Weight $(\mathrm{kg})$ & $74.0(62.9,87.0)$ & $71.2(60.2,83.6)$ & $<0.001^{*}$ \\
Deprivation score & $0.17(0.09,0.31)$ & $0.17(0.09,0.30)$ & 0.214 \\
Sex & & & $<0.001^{*}$ \\
Male & $9,763(97.1 \%)$ & $291(2.9 \%)$ & \\
Female & $14,677(93.9 \%)$ & $959(6.1 \%)$ & \\
Ethnicity & & & \\
Asian & $1,601(95.5 \%)$ & $75(4.5 \%)$ & \\
Black & $755(95.7 \%)$ & $34(4.3 \%)$ & \\
Mixed & $228(94.6 \%)$ & $13(5.4 \%)$ & \\
Other & $414(97 \%)$ & $13(3 \%)$ & \\
White & $19,722(94.8 \%)$ & $1,079(5.2 \%)$ & \\
Comorbidities & & & \\
0 & $12,888(96.5 \%)$ & $474(3.5 \%)$ & \\
1 & $4,735(95.2 \%)$ & $239(4.8 \%)$ & \\
$2+$ & $6,822(92.7 \%)$ & $537(7.3 \%)$ & \\
Admissions prior to first reported allergy & & \\
No & $4,871(96.8 \%)$ & $161(3.2 \%)$ & \\
Yes & $19,574(94.7 \%)$ & $1,089(5.3 \%)$ & \\
\hline Continuous data reported as: median (lower quartile, upper quartile), with \\
$p$ value from Mann-Whitney test & & \\
Categorical data reported as: $n(\%)$, with $p$ value from Fisher's exact test \\
* Significant at $p<0.05$ & & \\
& &
\end{tabular}

significantly associated with MDIS, contrary to the findings of the univariable analysis. This is likely related to the fact that patients with comorbidities tended to be older (median age: single comorbidity $=50$ years, multiple comorbidities $=66$ years, $p<0.001)$, and that male patients were heavier (median weight: male $=81 \mathrm{~kg}$, female $=69 \mathrm{~kg}$, $p<0.001)$.

Univariable analysis showed MDIS was significantly more frequent in patients prescribed an antihistamine $(p<0.001)$, prednisolone $(p<0.001)$, hydrocortisone $(p<0.001)$, or an EpiPen ${ }^{\circledR}(p=0.002)$ compared to those without such prescriptions (see Table 3). There was no significant difference in MDIS cases in patients with either an atopic or psychological comorbidity compared to patients without ( $p=0.444$, and $p=0.951$, respectively).

Multivariable ORs were also calculated for selected antiallergic agents and specific comorbidities. MDIS was associated with the prescription of antihistamine (OR 1.86, $95 \%$ CI 1.59-2.17, $p<0.001$ ), EpiPen ${ }^{\circledR}$ (OR 2.36, $95 \%$ CI $1.02-5.46, p=0.046$ ), and prednisolone (OR 1.25, $95 \%$ CI $1.05-1.49, p=0.014)$; however, hydrocortisone prescriptions were not significant predictors of MDIS in the multivariable analysis $(p=0.628)$. This is likely related to correlations with antihistamine and prednisolone (Kendalls tau $b=0.35$ and 0.38 , respectively).
Table 2 Multivariable analysis of the effects of demographic factors on rates of multiple drug intolerance

\begin{tabular}{|c|c|c|}
\hline Factor & Odds ratio $(95 \% \mathrm{CI})$ & $p$ value \\
\hline Age (years) & & 0.716 \\
\hline$<45$ & - & - \\
\hline $45-64$ & $1.02(0.86-1.22)$ & 0.789 \\
\hline $65+$ & $1.07(0.90-1.27)$ & 0.436 \\
\hline Weight (kg) & & 0.364 \\
\hline$<50$ & $1.04(0.82-1.34)$ & 0.731 \\
\hline $50-85$ & - & - \\
\hline$>85$ & $0.90(0.77-1.05)$ & 0.185 \\
\hline Deprivation score & & 0.646 \\
\hline$<0.1$ & - & - \\
\hline $0.1-0.3$ & $0.96(0.83-1.12)$ & 0.634 \\
\hline$>0.3$ & $0.92(0.76-1.10)$ & 0.350 \\
\hline Sex & & $<0.001^{*}$ \\
\hline Female & $2.06(1.77-2.40)$ & $<0.001^{*}$ \\
\hline Male & - & - \\
\hline Ethnicity & & 0.133 \\
\hline White & - & - \\
\hline Asian & $0.87(0.66-1.13)$ & 0.295 \\
\hline Black & $0.76(0.51-1.13)$ & 0.174 \\
\hline Mixed & $1.11(0.60-2.07)$ & 0.736 \\
\hline Other & $0.66(0.35-1.26)$ & 0.209 \\
\hline Unspecified & $0.62(0.40-0.97)$ & 0.035 \\
\hline Comorbidities & & $<0.001^{*}$ \\
\hline None & - & - \\
\hline 1 & $1.26(1.05-1.52)$ & $0.012 *$ \\
\hline 2 or more & $1.91(1.64-2.22)$ & $<0.001^{*}$ \\
\hline \multicolumn{2}{|c|}{ Admissions prior to first allergy } & $<0.001 *$ \\
\hline No & - & - \\
\hline Yes & $1.59(1.30-1.94)$ & $<0.001 *$ \\
\hline
\end{tabular}

Results from a multivariable binary logistic regression

* Significant at $p<0.05$

Univariable analysis showed that, except for penicillins, MDIS cases were significantly more frequent in patients with a documented allergy to any of the drug groups listed $(p<0.001$ for all). However, the frequency of MDIS cases were similar between patients with a penicillin allergy and those without a penicillin allergy $(4.9$ vs. $4.8 \%$, $p=0.749$ ) (see Tables 4, 5).

ORs were also expressed for each drug or drug group as predictors for multiple drug intolerance (Fig. 1). Of the group of drugs investigated, cephalosporin and quinolone allergies were the most significant predictors of MDIS (OR 11.3 and 11.1, respectively). The data also showed that, after penicillin, the likelihood of developing MDIS was lowest in those allergic to peanuts and shellfish (OR 2.3 for both). Furthermore, ORs were significantly smaller in those allergic to aspirin (OR 2.6, $95 \%$ CI 2.3-3.0) compared to 
Table 3 Effects of selected drugs and comorbidities on rates of multiple drug intolerance

\begin{tabular}{|c|c|c|c|c|c|}
\hline \multirow[t]{2}{*}{ Factor } & \multicolumn{2}{|c|}{ Multiple drug intolerance } & \multirow[t]{2}{*}{$p$ value } & \multirow{2}{*}{$\begin{array}{l}\text { Multivariable odds } \\
\text { ratio }^{\text {a }}(95 \% \mathrm{CI})\end{array}$} & \multirow[t]{2}{*}{$p$ value } \\
\hline & No & Yes & & & \\
\hline Antihistamine prescribed & & & $<0.001 *$ & & $<0.001 *$ \\
\hline No $(N=21,846)$ & $20,945(95.9 \%)$ & $901(4.1 \%)$ & & - & - \\
\hline Yes $(N=3,849)$ & $3,500(90.9 \%)$ & $349(9.1 \%)$ & & $1.86(1.59-2.17)$ & $<0.001 *$ \\
\hline EpiPen prescribed & & & $0.002 *$ & & $0.046^{*}$ \\
\hline No $(N=25,647)$ & $24,405(95.2 \%)$ & $1,242(4.8 \%)$ & & - & - \\
\hline Yes $(N=48)$ & $40(83.3 \%)$ & $8(16.7 \%)$ & & $2.36(1.02-5.46)$ & $0.046^{*}$ \\
\hline Prednisolone prescribed & & & $<0.001 *$ & & $0.014 *$ \\
\hline No $(N=22,461)$ & $21,464(95.6 \%)$ & $997(4.4 \%)$ & & - & - \\
\hline Yes $(N=3,234)$ & $2,981(92.2 \%)$ & $253(7.8 \%)$ & & $1.25(1.05-1.49)$ & $0.014 *$ \\
\hline Hydrocortisone prescribed & & & $<0.001^{*}$ & & 0.628 \\
\hline No $(N=23,691)$ & $22,609(95.4 \%)$ & $1,082(4.6 \%)$ & & - & - \\
\hline Yes $(N=2,004)$ & $1,836(91.6 \%)$ & $168(8.4 \%)$ & & $1.05(0.85-1.31)$ & 0.628 \\
\hline Psychological comorbidity & & & 0.951 & & 0.589 \\
\hline No $(N=24,594)$ & $23,398(95.1 \%)$ & $1,196(4.9 \%)$ & & - & - \\
\hline Yes $(N=1,101)$ & $1,047(95.1 \%)$ & $54(4.9 \%)$ & & $0.91(0.65-1.28)$ & 0.589 \\
\hline Atopic comorbidity & & & 0.444 & & 0.903 \\
\hline No $(N=23,050)$ & $21,920(95.1 \%)$ & $1,130(4.9 \%)$ & & - & - \\
\hline Yes $(N=2,645)$ & $2,525(95.5 \%)$ & $120(4.5 \%)$ & & $1.01(0.81-1.26)$ & 0.903 \\
\hline
\end{tabular}

Categorical data reported as: $n(\%)$, with $p$ value from Fisher's exact test

* Significant at $p<0.05$

${ }^{a}$ From multivariable binary logistic regression, adjusting for all factors in Tables 1 and 2

patients allergic to other drugs such as opioids (OR 4.3, $95 \%$ CI 3.8-4.9), antihistamines (OR 5.1, $95 \%$ CI 3.9-6.6), and other NSAIDs (OR 5.4, $95 \%$ CI 4.7-6.2).

\section{Discussion}

This is the first large-scale UK study to look at the effects of demographics, medical history, and medication use on the rates of MDIS. The MDIS cohort was compared to patients with one or two documented drug allergies in order to ascertain factors linked to multiple drug intolerances. The majority of patients in the control group were assumed to represent a cohort with true type 1 IgE-mediated hypersensitivity reactions. As such, comparing the MDIS cohort to this group of patients enabled the identification of risk factors specifically for the development of multiple drug intolerance rather than single drug allergies in general.

Among the drug allergies investigated, 18 out of 19 drug groups were shown to be significant risk factors for MDIS, with quinolones, cephalosporins, tetracyclines, and ACEi being identified as the most significant predictors. "Other antibiotic" allergies also formed a significant risk factor for MDIS. This is likely to be a result of the study population we used, all of whom were inpatients tending to be prescribed a wide spectrum of antibiotics during their hospital stay. Interestingly, with the exception of ACEi, the most significant risk factors for MDIS were allergies to broadspectrum antibiotics prescribed for short-term use. This may suggest that mechanisms underlying MDIS occur with a short latency period.

Penicillin allergy is commonly reported among UK patients [16]. As such, we expected that a penicillin allergy may increase the risk of being intolerant to multiple drugs. Indeed, Smith et al. [17] found that a history of allergy to other drugs was almost three times as common in patients who were penicillin-allergic compared to those who were not [5]. However, we found that the frequency of MDIS cases did not differ significantly between patients with penicillin allergy compared to those without. The frequency of MDIS was greatest in those allergic to broadspectrum antibiotics and these drugs were also the most significant risk factors for MDIS. Penicillins, however, did not fit this trend. This could potentially be explained by the fact that reported penicillin allergies are likely to represent true IgE-mediated hypersensitivity reactions.

Consistent with previous studies [6, 18], we found that even after adjusting for all other demographics, female gender is a significant risk factor for MDIS. This finding 
Table 4 Effects of specific nonantibiotic drugs on rates of multiple drug intolerance

\begin{tabular}{|c|c|c|c|}
\hline \multirow[t]{2}{*}{ Drug class } & \multicolumn{2}{|c|}{ Multiple drug intolerance } & \multirow[t]{2}{*}{$p$ value } \\
\hline & No & Yes & \\
\hline ACEi & & & $<0.001^{\prime}$ \\
\hline No $(N=25,277)$ & $24,133(95.5 \%)$ & $1,144(4.5 \%)$ & \\
\hline Yes $(N=418)$ & $312(74.6 \%)$ & $106(25.4 \%)$ & \\
\hline Antihistamines & & & $<0.001^{*}$ \\
\hline No $(N=25,296)$ & $24,125(95.4 \%)$ & $1,171(4.6 \%)$ & \\
\hline Yes $(N=399)$ & $320(80.2 \%)$ & $79(19.8 \%)$ & \\
\hline Aspirin & & & $<0.001^{*}$ \\
\hline No $(N=23,198)$ & $22,210(95.7 \%)$ & $988(4.3 \%)$ & \\
\hline Yes $(N=2,497)$ & $2,235(89.5 \%)$ & $262(10.5 \%)$ & \\
\hline Latex & & & $<0.001^{*}$ \\
\hline No $(N=25,203)$ & $24,013(95.3 \%)$ & $1,190(4.7 \%)$ & \\
\hline Yes $(N=492)$ & $432(87.8 \%)$ & $60(12.2 \%)$ & \\
\hline Lipid regulators & & & $<0.001^{*}$ \\
\hline No $(N=25,356)$ & $24,180(95.4 \%)$ & $1,176(4.6 \%)$ & \\
\hline Yes $(N=339)$ & $265(78.2 \%)$ & $74(21.8 \%)$ & \\
\hline NSAIDs & & & $<0.001^{*}$ \\
\hline No $(N=24,143)$ & $23,177(96 \%)$ & $966(4 \%)$ & \\
\hline Yes $(N=1,552)$ & $1,268(81.7 \%)$ & $284(18.3 \%)$ & \\
\hline Opioids & & & $<0.001^{*}$ \\
\hline No $(N=22,531)$ & $21,719(96.4 \%)$ & $812(3.6 \%)$ & \\
\hline Yes $(N=3,164)$ & $2,726(86.2 \%)$ & $438(13.8 \%)$ & \\
\hline Paracetamol & & & $<0.001^{*}$ \\
\hline No $(N=25,424)$ & $24,225(95.3 \%)$ & $1,199(4.7 \%)$ & \\
\hline Yes $(N=271)$ & $220(81.2 \%)$ & $51(18.8 \%)$ & \\
\hline Peanuts & & & $<0.001^{*}$ \\
\hline No $(N=25,427)$ & $24,205(95.2 \%)$ & $1,222(4.8 \%)$ & \\
\hline Yes $(N=268)$ & $240(89.6 \%)$ & $28(10.4 \%)$ & \\
\hline Shellfish & & & $<0.001^{\prime}$ \\
\hline No $(N=25,404)$ & $24,184(95.2 \%)$ & $1,220(4.8 \%)$ & \\
\hline Yes $(N=291)$ & $261(89.7 \%)$ & $30(10.3 \%)$ & \\
\hline Other nonantibiotics & & & $<0.001^{\prime}$ \\
\hline No $(N=22,106)$ & $21,504(97.3 \%)$ & $602(2.7 \%)$ & \\
\hline Yes $(N=3,589)$ & $2,941(81.9 \%)$ & $648(18.1 \%)$ & \\
\hline
\end{tabular}

Categorical data reported as: $n(\%)$, with $p$ value from Fisher's exact test

* Significant at $p<0.05$

could be linked to gender differences in healthcare use. It is well known that women have higher healthcare utilisation than men [19]. Women are therefore liable to be exposed to a larger range of drugs, making them more likely to report drug allergies and be identified as intolerant.

MDIS patients were found to be significantly older than non-MDIS patients. Older patients are known to have more comorbidities and therefore likely to have greater exposure to drugs, which increases the likelihood of a reported adverse reaction. The fact that age was not significant when
Table 5 Effects of specific antibiotics on rates of multiple drug intolerance

\begin{tabular}{lccc}
\hline Drug class & \multicolumn{2}{c}{ Multiple drug intolerance } & \multirow{2}{*}{$p$ value } \\
\cline { 2 - 3 } & No & \\
\hline Cephalosporins & & $<0.001^{*}$ \\
No $(N=25,336)$ & $24,210(95.6 \%)$ & $1,126(4.4 \%)$ & \\
Yes $(N=359)$ & $235(65.5 \%)$ & $124(34.5 \%)$ & \\
Glycopeptides & & & $<0.001^{*}$ \\
No $(N=25,513)$ & $24,303(95.3 \%)$ & $1,210(4.7 \%)$ & \\
Yes $(N=182)$ & $142(78 \%)$ & $40(22 \%)$ & \\
Macrolides & & & $<0.001^{*}$ \\
No $(N=24,557)$ & $23,557(95.9 \%)$ & $1,000(4.1 \%)$ & \\
Yes $(N=1,138)$ & $888(78 \%)$ & $250(22 \%)$ & \\
Penicillins & & & \\
No $(N=11,982)$ & $11,405(95.2 \%)$ & $577(4.8 \%)$ & \\
Yes $(N=13,713)$ & $13,040(95.1 \%)$ & $673(4.9 \%)$ & \\
Quinolones & & & $<0.001^{*}$ \\
No $(N=25,447)$ & $24,283(95.4 \%)$ & $1,164(4.6 \%)$ & \\
Yes $(N=248)$ & $162(65.3 \%)$ & $86(34.7 \%)$ & \\
Tetracyclines & & & $<0.001^{*}$ \\
No $(N=25,378)$ & $24,218(95.4 \%)$ & $1,160(4.6 \%)$ & \\
Yes $(N=317)$ & $227(71.6 \%)$ & $90(28.4 \%)$ & \\
Trimethoprim/sulfonamides & & & $<0.001^{*}$ \\
No $(N=24,554)$ & $23,535(95.8 \%)$ & $1,019(4.2 \%)$ & \\
Yes $(N=1,141)$ & $910(79.8 \%)$ & $231(20.2 \%)$ & \\
Other antibiotics & & & \\
No $(N=25,175)$ & $24,078(95.6 \%)$ & $1,097(4.4 \%)$ & \\
Yes $(N=520)$ & $367(70.6 \%)$ & $153(29.4 \%)$ & \\
\hline Categotcal & & \\
\hline
\end{tabular}

Categorical data reported as: $n(\%)$, with $p$ value from Fisher's exact test

* Significant at $p<0.05$

included in a multivariable model with the presence of comorbidities, suggests that the presence of increased comorbidities with age, rather than age independently, is the more important predictor of MDIS. These findings support previous work carried out by Onder et al. [20], who highlighted the significance of multiple comorbidities as predictors of adverse drug reactions.

Although De Pasquale et al. [8] found an increased likelihood of somatisation in their MDIS cohort, we showed that rates of psychological comorbidities were similar between MDIS and non-MDIS patients. This disparity is likely to be caused by differences in methodology. We used physicianled diagnoses of psychological disorders, whereas De Pasquale et al. [8] used psycho-diagnostic questionnaires to evaluate patients. Somatisation disorders have recently been identified as key risk factors for MDIS [9]. These disorders are difficult to identify and diagnose in clinical practice. This may explain why the prevalence of psychological comorbidities was not high in our MDIS cohort. 


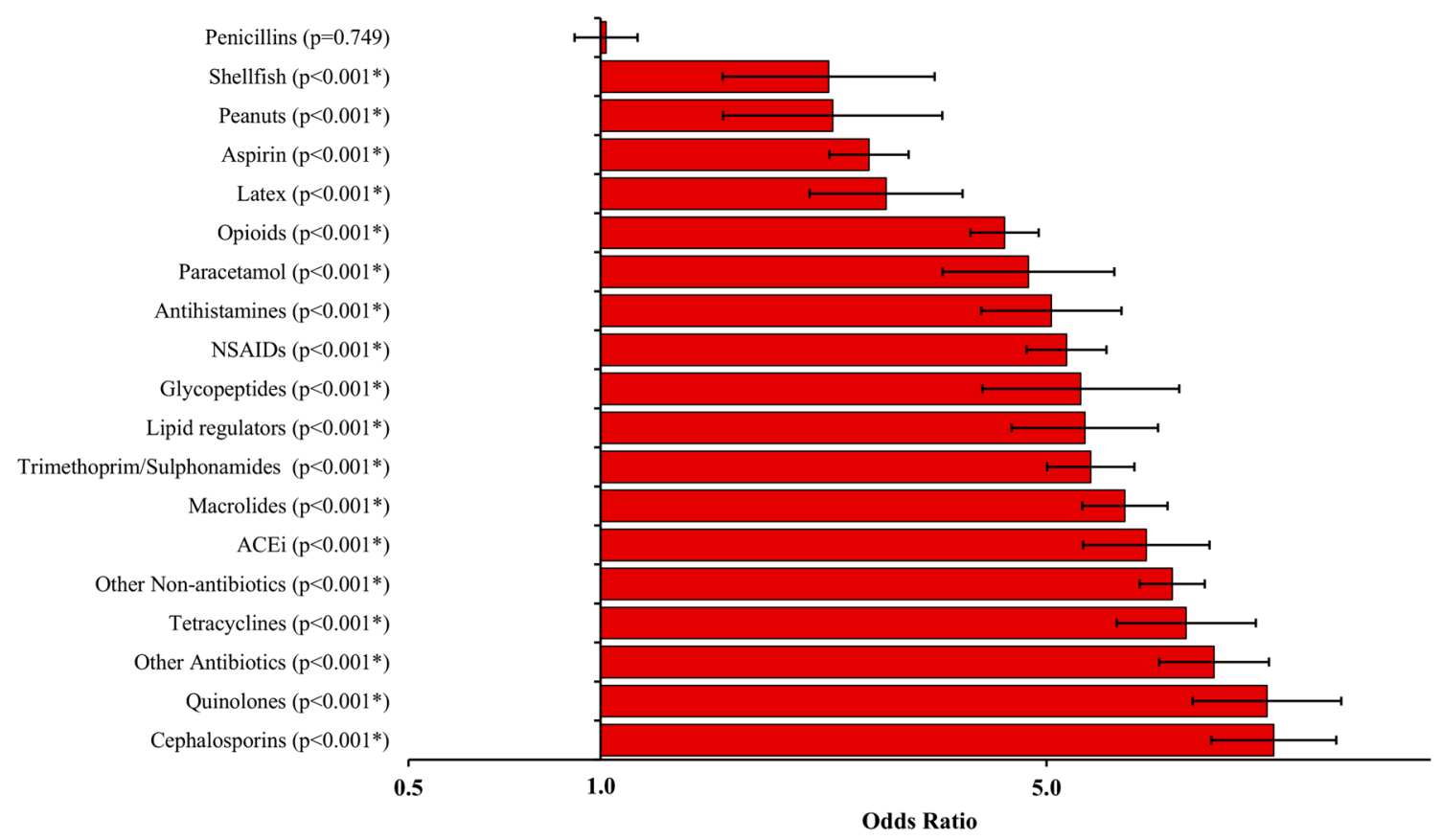

Fig. 1 Predictors of multiple drug intolerance

Immunological mechanisms may be more important than psychological factors in governing MDIS. We reported more cases of MDIS in those prescribed any antiallergic agent compared to those without such prescriptions. Such immunological mechanisms seem to differ from those involved in systemic drug sensitisations as we showed, like previous reports [18], that atopic comorbidities were not significant risk factors for MDIS.

In contrast to the work carried out by Macy and Ho [4], we found that patients with MDIS tended to be lighter in body weight. This disparity may be explained by selection bias, because it is possible that the heaviest patients would be the least likely to be weighed due to logistical difficulties. Although estimated weights were provided in some cases, these may have been highly inaccurate. Indeed, recent figures from the Health Survey for England 2011 suggest that women tend to underestimate their weights by $3.6 \mathrm{~kg}$ on average [21]. Women formed the majority of our study population and MDIS cohort. This may further explain why MDIS cases in our study had lower documented body weights than in previous reports [5].

We also found that weight as a factor was not a significant predictor for MDIS when adjusted for all other demographics, even though heavier patients tend to have multiple comorbidities and, as such, greater medication use $[22,23]$. It may be that multiple comorbidities represent the most important risk factor. Deprivation however, was not found to be a significant risk factor for MDIS.

We showed that the likelihood of MDIS is increased in those with documented allergies to different specific drug classes. This may not be surprising given the definition of multiple drug intolerance, because patients with an allergy to a single drug are more likely to meet the MDIS criteria compared to those without allergies. Importantly, we showed that the propensity for different drug allergies to increase the likelihood of MDIS varies and that penicillin allergies do not share this relationship.

\subsection{Limitations}

The dataset used for this study was based on documented allergies within the PICS system, reported by patients on admission to hospital. This was dependent on complete and accurate documentation by physicians. Some data were excluded owing to the absence of information (e.g., weight), which reduced the size of the dataset.

We are unable to state whether the allergies reported were true type I hypersensitivity reactions. Given that allergy testing is only recommended in patients who experience anaphylactic reactions to a drug, patients who report drug "allergies" are unlikely to have undergone allergy testing unless this manifested as a suspected anaphylaxis [2]. If allergy testing was carried out, however, such tests may have been conducted in hospitals different to that of our study site. Because UK hospitals do not share medical information across sites, use of allergy test data in our study would only represent patients investigated at our study site, potentially producing misleading results.

We used data that was derived from a secondary care setting and assumed that patients would have similar drug 
allergy reporting habits in primary care. An additional limitation is that prescribing guidelines might prevent the investigation of potential drug relationships. For example, it is well documented that cephalosporins should be avoided in those allergic to penicillins owing to the risk of cross reactivity. As such, patients with a reported penicillin allergy are less likely to be exposed to a cephalosporin and are therefore less likely to report an allergy to this drug class. Most antiallergic agents would have been prescribed in a primary care setting and antihistamines may have been taken over the counter; as such, it is likely that the prevalence of their use was underestimated in this study.

Finally, we calculated the deprivation scores based on household income, which may not fully reflect patient background and education.

\section{Conclusions}

We found the prevalence of MDIS to be greatest in female patients who have a number of documented comorbidities and who have both high healthcare (numerous hospital admissions) and medication use. After accounting for this, age, weight, ethnicity, and deprivation were not found to affect the likelihood of MDIS. Allergies to a broad spectrum of other drugs including nonpenicillin antibiotics were found to be significant risk factors for the development of MDIS, highlighting the potential for crossintolerance.

Acknowledgments The authors thank Mariam Afzal for data capture and Gavin Rudge for assistance with deprivation coding.

Funding This work was funded by the National Institute for Health Research (NIHR). Hisham M. R. B. Omer, James Hodson, Sarah K. Thomas, and Jamie J. Coleman are funded through the Collaborations for Leadership in Applied Health Research and Care for Birmingham and Black Country (CLAHRC-BBC) programme.

The views expressed in this publication are not necessarily those of the NIHR, the Department of Health, NHS Partner Trusts, Universities of Birmingham or CLAHRC-BBC Theme 9 Management/ Steering Group.

Competing interests All authors have completed the unified competing interest form at http://www.icmje.org/coi_disclosure.pdf (available on request from the corresponding author) and declare: Hisham M. R. B. Omer, James Hodson, Sarah K. Thomas, and Jamie J. Coleman had financial support in the form of a research grant from the National Institute for Health Research (NIHR) for the submitted work; no financial relationships with any organisations that might have an interest in the submitted work in the previous 3 years; no other relationships or activities that could appear to have influenced the submitted work.

Statement of contributions All authors had full access to all of the data (including statistical reports and tables) in the study and can take responsibility for the integrity of the data and the accuracy of the data analysis. All authors contributed to the writing of the manuscript, the interpretation of data, and approved the final version.

Open Access This article is distributed under the terms of the Creative Commons Attribution Noncommercial License which permits any noncommercial use, distribution, and reproduction in any medium, provided the original author(s) and the source are credited.

\section{References}

1. Sullivan TJ, Ong RC, Gilliam LK. Studies of the multiple drug allergy syndrome. J Allergy Clin Immunol. 1989;83:270.

2. National Institute for Health and Care Excellence. Drug allergy: diagnosis and management of drug allergy in adults, children and young people (CG183). London: National Institute for Health and Care Excellence; 2014.

3. Schiavino D, Nucera E, Roncallo C, Pollastrini E, De Pasquale T, Lombardo $\mathrm{C}$, et al. Multiple-drug intolerance syndrome: clinical findings and usefulness of challenge tests. Ann Allergy Asthma Immunol. 2007;99(2):136-42. doi:10.1016/S1081-1206(10) 60637-0.

4. Macy E, Ho NJ. Multiple drug intolerance syndrome: prevalence, clinical characteristics, and management. Ann Allergy Asthma Immunol. 2012;108(2):88-93. doi:10.1016/j.anai.2011.11.006.

5. Warrington R. Multiple drug allergy syndrome. Can J Clin Pharmacol. 2000;7(1):18-9.

6. Asero R, Tedeschi A, Lorini M, Caldironi G, Barocci F. Sera from patients with multiple drug allergy syndrome contain circulating histamine-releasing factors. Int Arch Allergy Immunol. 2003;131(3):195-200.

7. Asero R. Multiple drug allergy syndrome: a distinct clinical entity. Curr Allergy Asthma Rep. 2001;1(1):18-22. doi:10.1007/ s11882-001-0092-5.

8. De Pasquale T, Nucera E, Boccascino R, Romeo P, Biagini G, Buonomo A, et al. Allergy and psychologic evaluations of patients with multiple drug intolerance syndrome. Intern Emerg Med. 2012;7(1):41-7. doi:10.1007/s11739-011-0510-1.

9. Hassel JC, Danner D, Hassel AJ. Psychosomatic or allergic symptoms? High levels for somatization in patients with drug intolerance. J Dermatol. 2011;38(10):959-65. doi:10.1111/j. 1346-8138.2011.01249.x.

10. Häuser WEH, Enck P. Nocebo phenomena in medicine: their relevance in everyday clinical practice. Dtsch Arztebl Int. 2012;109(26):459-65.

11. Nightingale PG, Adu D, Richards NT, Peters M. Implementation of rules based computerised bedside prescribing and administration: intervention study. BMJ. 2000;320(7237):750-3.

12. Rudge GM, Mohammed MA, Fillingham SC, Girling A, Sidhu K, Stevens AJ. The combined influence of distance and neighbourhood deprivation on emergency department attendance in a large english population: a retrospective database study. PLoS One. 2013;8(7):e67943. doi:10.1371/journal.pone.0067943.

13. Office for National Statistics. National Statistics Postcode products: guidance and methodology. http://www.ons.gov.uk/ons/ guide-method/geography/products/postcode-directories/-nspp-/ index.html. Accessed 25 Aug 2013.

14. Noble M, McLennan D, Wilkinson K, Whitworth A, Barnes H, Dibben C. The English Indices of Deprivation 2007. London: Department for Communities and Local Government 2008. http:// webarchive.nationalarchives.gov.uk/+/http:/www.communities. gov.uk/communities/neighbourhoodrenewal/deprivation/depri vation07/. Accessed 25 Aug 2013. 
15. The Poverty Site. Key facts. The poverty site. http://www. poverty.org.uk/summary/key\%20facts.shtml. Accessed 25 Aug 2013.

16. Mirakian R, Ewan PW, Durham SR, Youlten LJF, Dugué P, Friedmann PS, et al. BSACI guidelines for the management of drug allergy. Clin Exp Allergy. 2009;39(1):43-61. doi:10.1111/j. 1365-2222.2008.03155.x.

17. Smith JW, Johnson JE, Cluff LE. Studies on the epidemiology of adverse drug reactions. N Engl J Med. 1966;274(18):998-1002. doi:10.1056/NEJM196605052741804.

18. Asero R. Detection of patients with multiple drug allergy syndrome by elective tolerance tests. Ann Allergy Asthma Immunol. 1998;80(2):185-8. doi:10.1016/S1081-1206(10)62953-5.

19. Bertakis KD, Azari R, Helms LJ, Callahan EJ, Robbins JA. Gender differences in the utilization of health care services. J Fam Pract. 2000;49(2):147-52.
20. Onder G, Petrovic M, Tangiisuran B, et al. Development and validation of a score to assess risk of adverse drug reactions among in-hospital patients 65 years or older: The GerontoNet ADR Risk Score. Arch Intern Med. 2010;170(13):1142-8. doi:10. 1001/archinternmed.2010.153.

21. Health and Social Care Information Centre. Health Survey for England-2011. Health, social care and lifestyles [NS]. http:// www.hscic.gov.uk/catalogue/PUB09300. Accessed 25 Aug 2013.

22. Guh DP, Zhang W, Bansback N, Amarsi Z, Birmingham CL, Anis AH. The incidence of co-morbidities related to obesity and overweight: a systematic review and meta-analysis. BMC Public Health 2009;8(88). doi:10.1186/1471-2458-9-88.

23. Kit BK, Ogden CL, Flegal KM. Prescription medication use among normal weight, overweight, and obese adults, United States, 2005-2008. Ann Epidemiol. 2012;22(2):112-9. doi:10. 1016/j.annepidem.2011.10.010. 